\title{
FACTORIZATION OF DIFFERENTIAL OPERATORS
}

\section{ANTON ZETTL}

Abstract. A necessary and sufficient condition for a differential operator of order $n$ to be factorable into a product of operators of orders $n-r$ and $r$, for any $0<r<n$, is given.

Consider differential operators of the form

$$
L y=s_{0} y^{(n)}+s_{1} y^{(n-1)}+\cdots+s_{n} y
$$

with $s_{i}$ continuous for $i=0, \cdots, n$ and $s_{0}(t) \neq 0$.

THEOREM. Suppose $L$ is an operator of order $n$ of type $\left({ }^{*}\right)$ and $0<r$ $<n$. Then there exist operators $P$ and $Q$ both of type $\left(^{*}\right)$ of orders $n-r$ and $r$ respectively such that $L=P Q$ on some interval $I$ if and only if there exist $r$ linearly independent solutions $y_{1}, \cdots, y_{r}$ of Ly=0 satisfying the condition that the Wronskian $W_{r}=W_{r}\left(y_{1} \cdots y_{r}\right) \neq 0$ on $I$.

Proof. Suppose $L=P Q$ where $Q$ has order $r$. Since any solution of $Q y=0$ is also a solution of $L y=0$, we can choose any $r$ linearly independent solutions of $Q y=0$ and condition $W_{r} \neq 0$ will be satisfied.

On the other hand, assume $L y_{i}=0$ for $i=1, \ldots, r$ and $W_{r}\left(y_{1} \cdots y_{r}\right) \neq 0$. Let

$$
Q y=\operatorname{det}\left[\begin{array}{cc}
y_{1} \cdots y_{r} & y \\
y_{1}^{\prime} \cdots y_{r}^{\prime} & y^{\prime} \\
y_{1}^{r} \cdots y_{r}^{r} & y^{r}
\end{array}\right]=q_{0} y^{(r)}+q_{1} y^{(r-1)}+\cdots+q_{r} y .
$$

Note that $q_{0}=W_{r}\left(y_{1} \cdots y_{r}\right) \neq 0$ and $q_{i} \in C^{n+1-r-i}$. A direct computation shows that the coefficients $p_{i}$ for $i=0, \cdots, n-r$ can be chosen successively so that the coefficients of $y^{(n)}, y^{(n-1)}, \cdots, y^{(n-r)}$ in the product $P Q y$ where $P y=p_{0} y^{n-r}+\cdots+p_{n-r} y$ are $s_{0}, s_{1}, \cdots$, $s_{r}$, respectively. Hence the operator $N=L-P Q$ is of order less than $r$. But $y_{1}, \cdots, y_{r}$ are linearly independent solutions of $N y=0$. Hence $N=0$ and $L=P Q$.

Although the above theorem may have been known to Ince-see

Received by the editors July 20,1970 .

AMS 1970 subject classifications. Primary 34A30, 34A05; Secondary 34A01.

Key words and phrases. Ordinary differential equations, factoring differential operators, Frobenius factorization, Polya's property " $W$ ", Wronskians, linear homogeneous differential equations. 
the discussion on pp. 119 and 126-127 in [1] - the author has not seen an explicit statement of it anywhere in the literature.

The conditions $W_{r} \neq 0$ for $r=1, \cdots, n-1$ are known to be necessary and sufficient for the factorability of an operator of type $\left(^{*}\right)$ into a "product" of first order operators-see [2].

\section{REFERENCES}

1. E. L. Ince, Ordinary differential equations, Dover, New York, 1944. MR 6, 65.

2. G. Polya, On the mean value theorem corresponding to a given linear homogeneous differential equation, Trans. Amer. Math. Soc. 24 (1922), 312-324.

Northern Illinois University, De Kalb, ILlinois 60115 